\title{
A LOSS-OF-ALLELES M:ODEL FOR THE EVOLUTION OF DISTYLY
}

\author{
GAYLE MUENCHOW \\ Department of Environmental, Population and Organismal Biology, \\ University of Colorado, Boulder, Colorado 80309, U.S.A.
}

Received 6.vii.81

\begin{abstract}
SUMMARY
Distyly is characterized by a diallelic incompatibility system. Crowe (1964) hypothesized that this has arisen by a loss of alleles from multiallelic systems. I have examined a mechanism for such a loss in a simulated population with sporophytic incompatibility and four alleles at the incompatibility locus. If a second locus is tightly linked to the incompatibility locus and a favoured new mutation spreads at this second locus, two of the four alleles at the incompatibility locus may be driven to extinction, leaving only the incompatibility allele originally linked to the favoured new mutation, and a second incompatibility allele that is dominant to the first. The two selection pressures responsible for this behaviour are the higher fitness of the new mutation at the second locus and the ever-acting selection for maximal cross-compatibility at the incompatibility locus. The implications of the model for the evolution of distyly are discussed.
\end{abstract}

\section{INTRODUCTION}

DISTYLY is a breeding system that usually involves both a sporophytically controlled diallelic incompatibility system and dimorphism in one or more of the following floral characters: style length, anther height, pollen size, pollen shape, exine structure and/or stigma surface structure. It is a system that has evolved convergently several times (Vuilleumier, 1967). Ganders (1979) has recently reviewed the subject. I wish to discuss the origin of the diallelic incompatibility system. This is thought to be fundamental to the rest of the pattern, the dimorphism arising either with or after the diallelic incompatibility but not preceding it (Baker, 1966; D. and B. Charlesworth, 1979).

There are two general hypotheses for the origin of this incompatibility system. The de novo hypothesis postulates that the system arose from a previous condition of self-compatibility. The Charlesworths (1979) have recently simulated this and in so doing assumed that the pollen and stylar incompatibility characters are controlled by the same two loci that control pollen size and style length respectively. The loss-of-alleles hypothesis, first stated by Crowe (1964), postulates that the two alleles arose from a previous multiallelic system by a loss of alleles. It is this hypothesis that I have simulated, and I assume the incompatibility is controlled by an $S$ locus such as is found in homomorphic taxa (for the purposes of the model I have called it $I$ ). An $S$ locus, because it has separable pollen and style regulatory parts (de Nettancourt, 1977), can in theory yield upon crossover the separation of pollen and stylar incompatibility observed in crossovers of the distyly supergene (Muenchow, 1981).

Fairly early in the study of incompatibility systems, Wright (1939) pointed out that individual selection for maximal cross-compatibility would 
be expressed as frequency dependent selection for incompatibility alleles. Rare alleles, since they seldom encounter an incompatible mating, will spread relative to more frequent alleles that suffer higher levels of crossincompatibility. Thus incompatibility alleles under Wright's model are not expected to be lost readily. This result has been cited as a reason for rejecting the loss-of-alleles hypothesis for the origin of distyly (Vuilleumier, 1967). But, as will be demonstrated below, it is possible to define a set of circumstances under which selection for maximal cross-compatibility-the same selective pressure that Wright studied-will act against the maintenance of incompatibility alleles, and alleles will go extinct. This behaviour and its possible applicability to the evolution of distyly are the subjects considered below.

\section{THE MODEL}

The model assumes a sporophytic incompatibility locus with four alleles that have the following relationships to each other in both the pollen and the carpel:

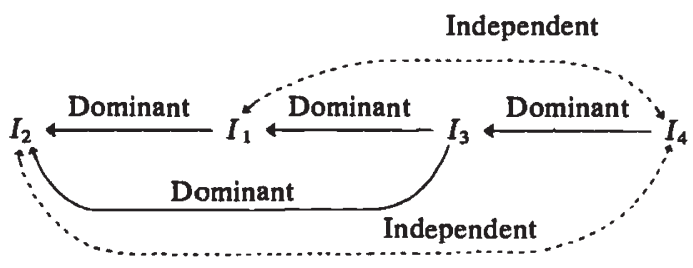

This is similar to patterns that have been found in sporophytic species (see Lewis, 1954 for review). I assume only four alleles, an underestimate of the number in natural populations, because this is a two-locus model and the number of genotypes increases geometrically with the number of alleles. By assuming just these four alleles and two at a second, $P$, locus, it is necessary at times to follow as many as 33 genotypes and 561 possible matings. Since successful mating is not random, but depends upon the identity of the sporophytic genotypes, it is not sufficient simply to follow allele and gamete frequencies. Furthermore, gametes with identical genetic constitutions may have different incompatibility phenotypes depending upon their sporophytic parentage. For example, the haploid pollen grain $P I_{1}$ has the incompatibility phenotype $I_{1}$ if it comes from the sporophytes $I_{1} I_{1}$ or $I_{1} I_{2}, I_{3}$ if it comes from the sporophyte $I_{1} I_{3}$, or $I_{1} I_{4}$ if it comes from the sporophyte $I_{1} I_{4}$. Thus, below, $I$ have called the sets of two linked loci "linkage groups" and not gamete types, since each linkage group can be composed of one or more phenotypic gamete types.

Much of the behaviour of the model is understandable once one appreciates the effect of cross-compatibility relationships on the frequencies of the various $I_{i} I_{j}$ genotypes. Table 1 lists all the possible $I_{i} I_{j}$ genotypes, their phenotypes, equilibrium frequencies and the other genotypes with which they are incompatible (incompatibility results when phenotypes match). With sporophytic incompatibility, dominance and independence can be expressed even though the pollen grain is haploid. The allele $I_{4}$, for example, 
TABLE 1

All the possible $\mathrm{I}_{\mathbf{i}} \mathrm{I}_{\mathbf{j}}$ genotypes of the four-allele model, the incompatibility phenotypes of each, the genotypes with which each is incompatible, and the equilibrium frequency of each as determined by the cross-compatibility relationships

$\begin{array}{cccc}\text { Genotype } & \begin{array}{c}\text { Pollen/Pistil } \\ \text { phenotype }\end{array} & \begin{array}{c}\text { Incompatible genotypes } \\ \text { Equilibrium } \\ \text { frequency }\end{array} \\ I_{1} I_{1} & 1 & \text { Self, } I_{1} I_{2}, I_{1} I_{4} & 0.024 \\ I_{1} I_{2} & 1 & \text { Self, } I_{1} I_{1}, I_{1} I_{4} & 0 \cdot 240 \\ I_{1} I_{3} & 3 & \text { Self, } I_{2} I_{3}, I_{3} I_{3} & 0.096 \\ I_{1} I_{4} & 1 \text { and } 4 & \text { Self, } I_{1} I_{1}, I_{1} I_{2}, I_{2} I_{4}, I_{3} I_{4} & 0.032 \\ I_{2} I_{2} & 2 & \text { Self, } I_{2} I_{4} & 0.254 \\ I_{2} I_{3} & 3 & \text { Self, } I_{1} I_{3}, I_{3} I_{3} & 0.244 \\ I_{2} I_{4} & 2 \text { and } 4 & \text { Self, } I_{1} I_{4}, I_{2} I_{2}, I_{3} I_{4} & 0.066 \\ I_{3} I_{3} & 3 & \text { Self, } I_{1} I_{3}, I_{2} I_{3} & 0.009 \\ I_{3} I_{4} & 4 & \text { Self, } I_{1} I_{4}, I_{2} I_{4} & 0.035\end{array}$

is either independent or dominant to the other alleles-it never "hides" by being recessive. Thus no $I_{4}$-carrying genotype can fertilize another $I_{4}$-carrying genotype, making it impossible to form the $I_{4} I_{4}$ genotype. Conversely, the $I_{2}$ allele is recessive to both $I_{1}$ and $I_{3}$-it "hides" in several genotypes. Thus there are rather few genotypes with which $I_{2}$-carrying genotypes cannot cross. The equilibrium frequencies of the various genotypes, calculated here by simulating mating without selection, reflect this cross-compatibility. $I_{4}$-carrying genotypes are maintained at fairly low levels, $I_{2}$-carrying genotypes at much higher levels, the others in between.

This model pushes the genotypes out of the cross-compatibility equilibrium of table 1 by assuming the allele $I_{1}$ is closely linked to a favourable new mutation $P^{*}$. As $P^{*}$ spreads it will tend to pull $I_{1}$ to a greater frequency. Since the favoured genotype $P^{*} P^{*}$ will almost always occur as the two-locus genotype $P^{*} I_{1} P^{*} I_{1}$, the selection at the $P$ locus will secondarily cause the selection for maximal cross-compatibility at the $I$ locus to take the form of selection for cross-compatibility with the now more common $I_{1} I_{1}$ genotype. From table 1 one sees that the genotypes $I_{1} I_{2}$ and $I_{1} I_{4}$ are incompatible with $I_{1} I_{1}$, so one might predict that $I_{2}$ and $I_{4}$ will decrease in frequency as $I_{1}$ increases. This happens, and in fact the alleles $I_{2}$ and $I_{4}$ can go extinct. The simulations below detail the requirements for their extinction.

For the model it is necessary only that $P$ be a mutable locus, a new mutation $P^{*}$ of which is selectively favoured and linked to $I_{1}$. Biological interpretations of the $P$ locus are discussed below. With the addition of the $P$ locus represented by the wild type allele $P$ and the new mutation $P^{*}$, the nine genotypes of table 1 become thirty-three genotypes:

\begin{tabular}{|c|c|c|c|c|c|}
\hline $\begin{array}{l}P I_{1} P I_{1} \\
P I_{1} P^{*} I_{1} \\
P^{*} I_{1} P^{*} I_{1}\end{array}$ & $\begin{array}{l}P I_{1} P I_{2} \\
P^{*} I_{1} P I_{2} \\
P I_{1} P^{*} I_{2} \\
P^{*} I_{1} P^{*} I_{2}\end{array}$ & $\begin{array}{l}P I_{1} P I_{3} \\
P^{*} I_{1} P I_{3} \\
P I_{1} P^{*} I_{3} \\
P^{*} I_{1} P^{*} I_{3}\end{array}$ & $\begin{array}{l}P I_{1} P I_{4} \\
P^{*} I_{1} P I_{4} \\
P I_{1} P^{*} I_{4} \\
P^{*} I_{1} P^{*} I_{4}\end{array}$ & $\begin{array}{l}P_{2} P_{2} \\
P^{*} I_{2} P I_{2} \\
P^{*} I_{2} P^{*} I_{2}\end{array}$ & $\begin{array}{l}P I_{2} P I_{3} \\
P^{*} I_{2} P I_{3} \\
P I_{2} P^{*} I_{3} \\
P^{*} I_{2} P^{*} I_{3}\end{array}$ \\
\hline $\begin{array}{l}P I_{2} P I_{4} \\
P^{*} I_{2} P I_{4} \\
P I_{2} P^{*} I_{4} \\
P^{*} I_{2} P^{*} I_{4}\end{array}$ & $\begin{array}{l}P_{3} P_{3} \\
P^{*} I_{3} P I_{3} \\
P^{*} I_{3} P^{*} I_{3}\end{array}$ & $\begin{array}{l}P_{3} P I_{4} \\
P^{*} I_{3} P I_{4} \\
P I_{3} P^{*} I_{4} \\
P^{*} I_{3} P^{*} I_{4}\end{array}$ & & & \\
\hline
\end{tabular}


The simulations all begin with predominantly $P I_{i} P I_{j}$ genotypes, usually in frequencies close to the cross-compatibility equilibrium frequencies for the $I$ locus. (I have run a number of simulations with the initial genotype frequencies well away from their equilibrium frequencies and have never found that to make any difference. The cross-compatibility equilibrium frequencies are largely re-established within ten to twenty generations and the simulations proceed from there.) $P^{*}$ is initially represented only by a low frequency of the genotype $P^{*} I_{1} P I_{2}$. The other $P^{*} I_{1}$-carrying genotypes are quickly formed by mating; the $P^{*} I_{2-}, P^{*} I_{3}$ - and $P^{*} I_{4}$-carrying genotypes depend upon recombination for their formation.

$P^{*}$ is given a selective advantage. Where $P^{*}$ is dominant, the genotypes $P^{*} P^{*}$ and $P^{*} P$ have a fitness of $(1+S)$ relative to 1.0 for $P P$; where $P^{*}$ is incompletely dominant, $P^{*} P^{*}$ has a fitness of $(1+S)$ and $P^{*} P$ has a fitness $(1+(S / 2))$ relative to 1.0 for $P P$; and where $P^{*}$ is recessive, $P^{*} P^{*}$ has a fitness of $(1+S)$ relative to $1 \cdot 0$ for both $P^{*} P$ and $P P$. Each generation, the $P^{*}$-carrying genotypes are increased in frequency in proportion to their fitnesses. Pollinations occur in proportion to the frequencies of the genotypes, but fertilizations depend upon the compatibility relationships between the genotypes involved. Each simulation charts the increase in frequency of $P^{*}$, any effect that may have on the frequency of $I_{1}$, and any further effect a change in the frequency of $I_{1}$ has on the frequencies of $I_{2}$, $I_{3}$, and $I_{4}$. The programs are in Fortran IV and were run on a CYBER 170 series computer at the University of Colorado.

There are three important variables that determine the outcome of a simulation-(a) the selective advantage of $P^{*},(\mathrm{~b})$ the recombination rate between the $P$ and $I$ loci, and (c) the likelihood that the rare recombinant groups $P^{*} I_{2}, P^{*} I_{3}$ and $P^{*} I_{4}$ are randomly lost. The role of the selective advantage of $P^{*}$ is first elucidated deterministically; the stochastic program then considers the roles of the other two.

\section{THE DETERMINISTIC PROGRAM}

In a deterministic program no genotype is ever lost randomly. In this case that means that the recombinant linkage groups $P^{*} I_{2}, P^{*} I_{3}$, and $P^{*} I_{4}$ will always become established in a population, even if the recombination rates are such that each recombinant group spends tens or hundreds of generations at extremely low frequencies. Thus if the recombination rate is greater than zero, the only equilibrium will be that at which $P^{*}$ and $I$ are no longer at linkage disequilibrium, $P^{*}$ has become fixed, and the various $I_{i} I_{j}$ genotypes have regained their cross-compatibility equilibrium frequencies as listed in table 1 . To study any other behaviour from the model requires assuming that the recombination rate is zero, which $I$ have done in all the deterministic simulations. That this requirement is an artifact caused by prohibiting any random loss of rare types will be demonstrated below.

Since the recombination rate is set at zero, $P^{*}$ can be associated only with the allele $I_{1}$. This linkage group, $P^{*} I_{1}$, rapidly replaces $P I_{1}$ when $P^{*}$ has a selective advantage, leaving only the nine genotypes listed in the heading of table 2 . Table 2 records the equilibrium frequency of each genotype as a function of the selective advantage of $P^{*}$. Each may be compared to the equilibrium frequency that it would have under 


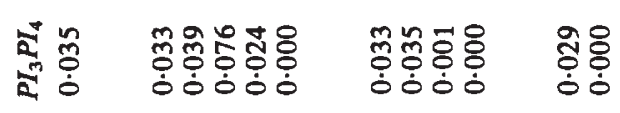

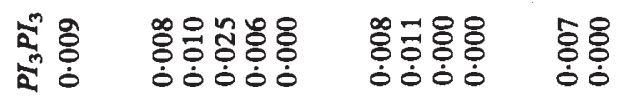

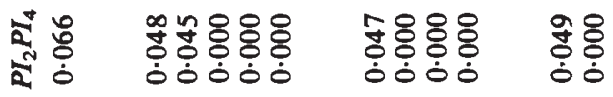

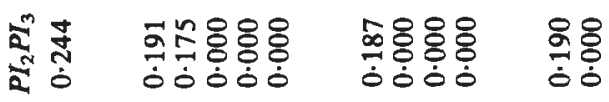

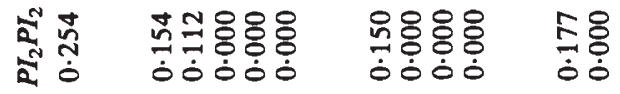

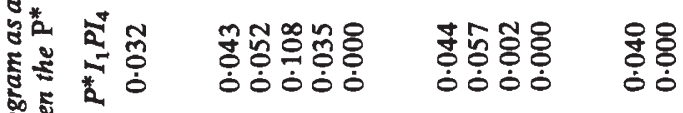

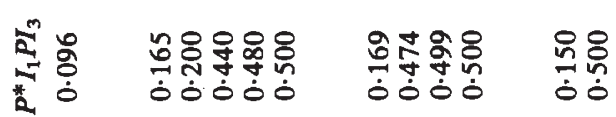

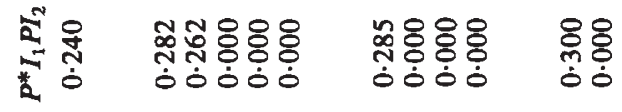

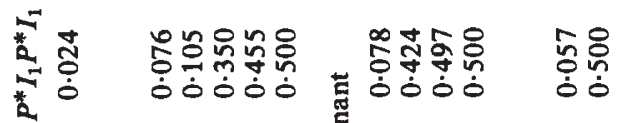

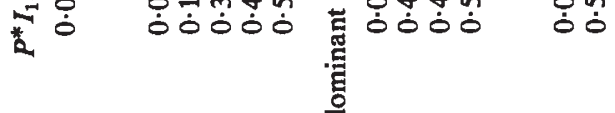

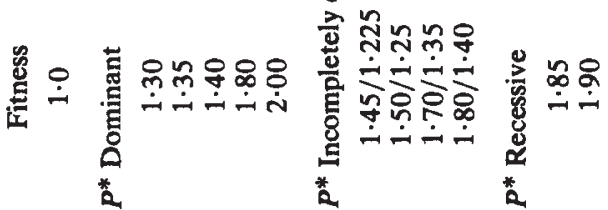


cross-compatibility considerations alone (table 1 and the first line of table 2). As table 2 shows, the selective advantage of $P^{*}$ affects the equilibrium frequencies. For the smaller selective advantages shown in table 2 , the advantage of $P^{*}$ merely pulls the equilibrium frequencies of the $P^{*}$-carrying genotypes to somewhat higher levels than would exist under cross-compatibility considerations alone. When the selective advantage of $P^{*}$ is greater than a certain critical value, however, the equilibrium frequencies of the genotypes are radically different. In particular, one or two of the four incompatibility alleles go extinct and the equilibrium genotype frequencies shift such that two genotypes come strongly to predominate-the genotypes $P^{*} I_{1} P^{*} I_{1}$ and $P^{*} I_{1} P I_{3}$, where $I_{3}$ is dominant to $I_{1}$. This is the distyly pattern.

The distyly pattern is elicited here by the mutual effect of two selective pressures-the advantage of $P^{*}$ and the ever-acting selection for maximal cross-compatibility. The advantage of $P^{*}$ causes the $P^{*}$-carrying genotypes to become more common. Simultaneously, selection for maximal crosscompatibility causes those $P^{*}$-carrying genotypes that cross well with each other to become more common and those that suffer high levels of crossincompatibility to become rarer, even to the point of extinction. This is the basis of the statement above that selection for maximal cross-compatibility can act under some circumstances against the maintenance of incompatibility alleles.

From table 2, the critical fitnesses (relative to $1 \cdot 0$ ) above which the distyly pattern emerges are as follows: when $P^{*}$ is dominant the critical fitness lies between 1.35 and 1.40 ; when $P^{*}$ is incompletely dominant, it lies between $1 \cdot 45 /\left(1.225\right.$ for the heterozygote $\left.P^{*} P\right)$ and $1 \cdot 50 /(1 \cdot 25$ for the $P^{*} P$ heterozygote); and when $P^{*}$ is recessive, it lies between 1.85 and 1.90 .

\section{THE STOCHASTIC PROGRAM}

In the stochastic program, recombination does occur between the $P$ and $I$ loci. When $P^{*}$ becomes linked to an $I$ allele other than $I_{1}$, the selective advantage of $P^{*}$ will tend to prevent the loss of that $I$ allele. Thus recombination acts counter to the behaviour under investigation here. The question posed by the stochastic program, then, is whether, and under what conditions, effective recombination can be too slow to prevent the loss of $I$ alleles. Effective recombination is determined both by the actual event of recombination and by the successful spread of the new recombinant types-here the recombinant types are $P^{*} I_{2}, P^{*} I_{3}$, and $P^{*} I_{4}$. Their successful spread is hindered by random death.

In particular, the stochastic program calculates the frequency of $P^{*} I_{2}$. It then asks whether that frequency is greater than or less than the number $5 / 2 N$, where $N$ is the population size. In other words it asks whether there are more or fewer than the equivalent of five individuals in the population heterozygous for $P^{*} I_{2}$. If there are more than five individuals, it assumes that $P^{*} I_{2}$ is well established and it lets it spread deterministically. If there are fewer than five individuals, however, the program generates a random number between zero and one. If that number is greater than a stated cutoff, all $P^{*} I_{2}$-carrying individuals survive and reproduce; if the number is less than the cutoff, all $P^{*} I_{2}$-carrying individuals fail to reproduce. The cutoff point is the probability of random death. The program then repeats 
this process for the recombinants, $P^{*} I_{3}$ and $P^{*} I_{4}$. Low recombination rates, small populations and populations with high levels of random death should tend to allow the loss of $I$ alleles.

Each of these stochastic simulations is a race. Linkage of $P^{*}$ to $I_{1}$ creates a situation in which $I_{3}$ is secondarily selected because of its relative cross-compatibility with $I_{1}$-bearing genotypes. High fitness of $P^{*}$ makes this evolutionary sequence, with the loss of $I$ alleles, occur quickly. Linkage

\section{TABLE 3}

From the stochastic program, the quasi-equilibrium frequencies, i.e., frequencies that have remained unchanged for at least 800 generations, of $\mathrm{P}^{*}, \mathrm{I}_{1} \mathrm{I}_{1}$ and $\mathrm{I}_{1} \mathrm{I}_{3}$ as a function of the selective advantage of $\mathrm{P}^{*}$, assuming the recombination rate between the $\mathrm{P}$ and $\mathrm{I}$ loci is $10^{-4}$, the population size is $10^{4}$ and the probability of random death is 0.5 . Each line represents a different simulation with a different sequence of random survival/death decisions
Fitness
Quasi-equilibrium frequencies$$
I_{1} I_{1}
$$$$
I_{1} I_{3}
$$
Comment

$P^{*}$ Dominant:

$$
P^{*}
$$

\begin{tabular}{|c|c|c|c|c|}
\hline \multirow[b]{2}{*}{ Fitness } & \multicolumn{3}{|c|}{ Quasi-equilibrium frequencies } & \multirow[b]{2}{*}{ Comment } \\
\hline & $P^{*}$ & $I_{1} I_{1}$ & $I_{1} I_{3}$ & \\
\hline \multicolumn{5}{|l|}{$P^{*}$ Dominant: } \\
\hline 1.40 & $0 \cdot 821$ & 0.024 & 0.090 & $\begin{array}{l}\text { No alleles lost; } P^{*} \text { recombines with } I_{2} \text { but } \\
\text { not } I_{3} \text { or } I_{4}\end{array}$ \\
\hline & $\begin{array}{l}0.821 \\
0.625\end{array}$ & $\begin{array}{l}0.024 \\
0.350\end{array}$ & $\begin{array}{l}0.090 \\
0.440\end{array}$ & $\begin{array}{l}\text { Same as above } \\
I_{2} \text { lost; } P^{*} \text { does not recombine }\end{array}$ \\
\hline $1 \cdot 50$ & $\begin{array}{l}1 \cdot 0 \\
0.830 \\
0.832\end{array}$ & $\begin{array}{l}0 \cdot 192 \\
0 \cdot 192 \\
0 \cdot 024\end{array}$ & $\begin{array}{l}0 \cdot 387 \\
0 \cdot 387 \\
0.089\end{array}$ & $\begin{array}{l}I_{2} \text { lost; } P^{*} \text { recombines with } I_{3} \text { and } I_{4} \\
I_{2} \text { lost; } P^{*} \text { recombines with } I_{3} \text { but not } I_{4} \\
I_{4} \text { lost; } P^{*} \text { recombines with } I_{2} \text { but not } I_{3}\end{array}$ \\
\hline 1.65 & $\begin{array}{l}0.682 \\
0.682 \\
0.833\end{array}$ & $\begin{array}{l}0.418 \\
0.418 \\
0.024\end{array}$ & $\begin{array}{l}0.465 \\
0.465 \\
0.089\end{array}$ & $\begin{array}{l}I_{2} \text { lost; } P^{*} \text { does not recombine } \\
\text { Same as above } \\
I_{4} \text { lost; } P^{*} \text { recombines with } I_{2} \text { but not } I_{3}\end{array}$ \\
\hline $1 \cdot 80$ & $\begin{array}{l}1 \cdot 0 \\
0 \cdot 712 \\
0 \cdot 712\end{array}$ & $\begin{array}{l}0 \cdot 192 \\
0 \cdot 454 \\
0 \cdot 454\end{array}$ & $\begin{array}{l}0.386 \\
0 \cdot 480 \\
0.480\end{array}$ & $\begin{array}{l}I_{2} \text { lost; } P^{*} \text { recombines with } I_{3} \text { and } I_{4} \\
I_{2} \text { lost; } P^{*} \text { does not recombine } \\
\text { Same as above }\end{array}$ \\
\hline $2 \cdot 00$ & $\begin{array}{l}1 \cdot 0 \\
0 \cdot 750 \\
0 \cdot 750\end{array}$ & $\begin{array}{l}0 \cdot 192 \\
0 \cdot 500 \\
0 \cdot 500\end{array}$ & $\begin{array}{l}0.386 \\
0 \cdot 500 \\
0 \cdot 500\end{array}$ & $\begin{array}{l}I_{2} \text { lost; } P^{*} \text { recombines with } I_{3} \text { and } I_{4} \\
I_{2} \text { and } I_{4} \text { lost; } P^{*} \text { does not recombine } \\
\text { Same as above }\end{array}$ \\
\hline \multicolumn{5}{|c|}{$P^{*}$ Incompletely dominant: } \\
\hline $1 \cdot 55 / 1 \cdot 275$ & $\begin{array}{l}1 \cdot 0 \\
1 \cdot 0 \\
0 \cdot 907\end{array}$ & $\begin{array}{l}0 \cdot 024 \\
0 \cdot 192 \\
0 \cdot 334\end{array}$ & $\begin{array}{l}0.090 \\
0.387 \\
0.450\end{array}$ & $\begin{array}{l}I_{4} \text { lost; } P^{*} \text { recombines with } I_{2} \text { and } I_{3} \\
I_{2} \text { lost; } P^{*} \text { recombines with } I_{3} \text { and } I_{4} \\
I_{2} \text { lost; } P^{*} \text { recombines with } I_{3} \text { but not } I_{4}\end{array}$ \\
\hline $1 \cdot 70 / 1 \cdot 35$ & $\begin{array}{l}0.930 \\
0.930 \\
0.930\end{array}$ & $\begin{array}{l}0 \cdot 375 \\
0 \cdot 375 \\
0 \cdot 375\end{array}$ & $\begin{array}{l}0.464 \\
0.464 \\
0.464\end{array}$ & $\begin{array}{l}I_{2} \text { lost; } P^{*} \text { recombines with } I_{3} \text { but not } I_{4} \\
\text { Same as above } \\
\text { Same as above }\end{array}$ \\
\hline $2 \cdot 00 / 1 \cdot 50$ & $\begin{array}{l}0.976 \\
0.976 \\
1 \cdot 0\end{array}$ & $\begin{array}{l}0.457 \\
0.457 \\
0.500\end{array}$ & $\begin{array}{l}0489 \\
0.489 \\
0 \cdot 500\end{array}$ & $\begin{array}{l}I_{2} \text { lost; } P^{*} \text { recombines with } I_{3} \text { but not } I_{4} \\
\text { Same as above } \\
I_{2} \text { and } I_{4} \text { lost; } P^{*} \text { recombines with } I_{3}\end{array}$ \\
\hline $2 \cdot 20 / 1 \cdot 60$ & $\begin{array}{l}1 \cdot 0 \\
1 \cdot 0 \\
1 \cdot 0\end{array}$ & $\begin{array}{l}0 \cdot 024 \\
0 \cdot 500 \\
0 \cdot 500\end{array}$ & $\begin{array}{l}0.089 \\
0.500 \\
0.500\end{array}$ & $\begin{array}{l}I_{4} \text { lost; } P^{*} \text { recombines with } I_{2} \text { and } I_{3} \\
I_{2} \text { and } I_{4} \text { lost; } P^{*} \text { recombines with } I_{3} \\
\text { Same as above }\end{array}$ \\
\hline \multicolumn{5}{|l|}{$P^{*}$ Recessive: } \\
\hline 1.90 & $\begin{array}{l}1 \cdot 0 \\
1 \cdot 0 \\
1 \cdot 0\end{array}$ & $\begin{array}{l}0.024 \\
0 \cdot 024 \\
0.024\end{array}$ & $\begin{array}{l}0.089 \\
0.089 \\
0.089\end{array}$ & $\begin{array}{l}I_{4} \text { lost; } P^{*} \text { recombines with } I_{3} \text { and } I_{4} \\
\text { Same as above } \\
\text { Same as above }\end{array}$ \\
\hline $2 \cdot 00$ & $\begin{array}{l}1 \cdot 0 \\
1 \cdot 0 \\
1 \cdot 0 \\
1 \cdot 0\end{array}$ & $\begin{array}{l}0.024 \\
0.500 \\
0 \cdot 500 \\
0.500\end{array}$ & $\begin{array}{l}0.090 \\
0.500 \\
0.500 \\
0.500\end{array}$ & $\begin{array}{l}I_{4} \text { lost; } P^{*} \text { recombines with } I_{3} \text { and } I_{4} \\
I_{2} \text { and } I_{4} \text { lost; } P^{*} \text { recombines with } I_{3} \\
\text { Same as above } \\
\text { Same as above }\end{array}$ \\
\hline
\end{tabular}




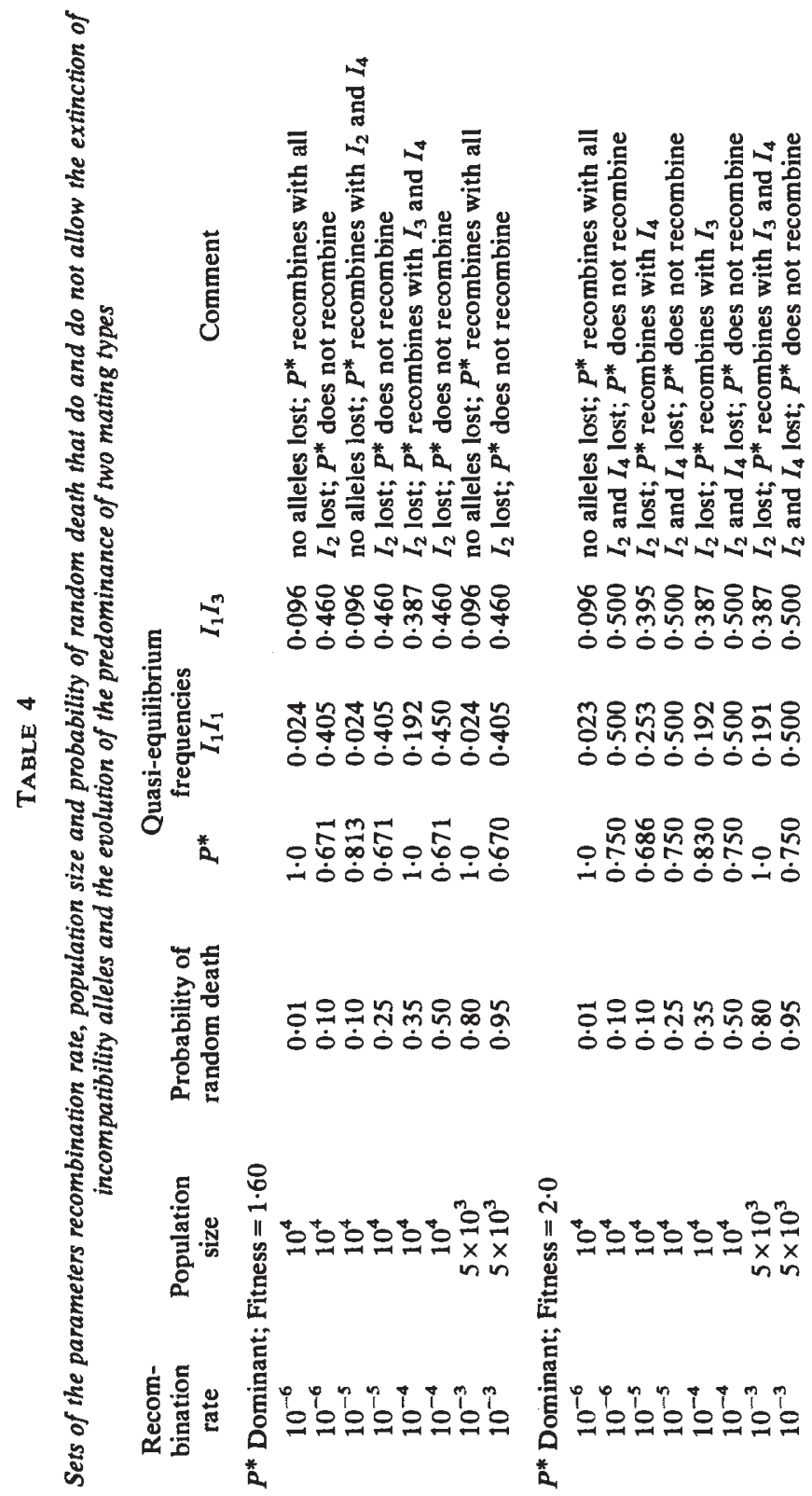


of $P^{*}$ to a second $I$ allele before $I$ alleles are lost will prevent loss of the $I$ allele now linked to $P^{*}$ and prevent the loss of one or both of the other two $I$ alleles as well. Tables 3 and 4 investigate the magnitudes of fitness and effective recombination respectively that allow the loss of $I$ alleles. Table 3 varies the fitness advantage of $P^{*}$ but holds the elements of effective recombination constant by holding the population size at $10^{4}$, the chromosomal recombination rate at $10^{-4}$ and the probability of random death at 0.5 . Random numbers generated each generation determine whether the rare recombinants survive. The sequence of these is important to the outcome, since the whole evolution may occur rapidly. There is usually a span of about 100 generations during which $I$ allele frequencies are high enough (before decreasing to extinction or to low equilibria) that chromosomal recombination is relatively frequent and the chances of establishment of a new recombinant are correspondingly relatively good. Thus the particular sequence of random survival/death decisions during these 100 generations can be critical to the simulation's outcome. In other words, the outcome in any one population depends to a great extent on that population's own history.

Each line in table 3 represents a different simulation and a different sequence of random numbers. The effect of the sequences is immediately apparent. At any fitness value, loss of $I$ alleles may or may not occur, depending mostly upon "runs of luck" during the more critical phases of the evolutionary change, though at higher fitness values the loss of $I$ alleles is more common. Table 3 lists all the simulations done under the stated conditions, not a selected set of them. Though the loss of $I$ alleles does not always occur, it commonly occurs.

$P^{*}$, the favoured allele, does not necessarily go to fixation, especially when it is dominant. This behaviour is discussed below.

In table 4 the fitness of $P^{*}$ and the sequence of random survival/death decisions are held constant in order best to examine the effects of various magnitudes of chromosomal recombination rate, population size and probability of random death. The pattern in table 4 is the expected one. In order to lose $I$ alleles, as recombination rates rise, probabilities of random death must also rise, thus keeping effective recombination low. From table 4 , loss of $I$ alleles despite chromosomal recombination rates of $10^{-6}$ or $10^{-5}$ (or those same rates of mutation at the $I$ locus) can occur in a population of $10^{4}$ with a 0.25 probability of random death, reasonable biological values for those parameters. Loss of $I$ alleles despite a chromosomal recombination rate of $10^{-3}$ requires lower population sizes and much higher probabilities of random death. Chromosomal recombination rates higher than $10^{-3}$ are apt to prevent the loss of $I$ alleles under any biologically realistic assumptions of population size and probability of random death.

\section{Discussion}

Individual selection for maximal cross-compatibility causes incompatibility genotypes to reach rapidly, and then to maintain, an equilibrium set of frequencies such that each genotype suffers the same level of crossincompatibility. If there is no second pressure perturbing the system, the selection for maximal cross-compatibility favours rare alleles, which means that functional new incompatibility mutations are favoured and alleles are 
not expected to be lost except by random extinction (genetic drift). When incompatibility genotypes are perturbed and kept away from this equilibrium by a second pressure-here, linkage of one allele to a favourable new mutation at a second locus-the continuing selection for maximal cross-compatibility will tend to hold the system near the unperturbed equilibrium when the second pressure is not too great. But the important result of the above model is that, given strong enough pressure away from the original equilibrium, selection for maximal cross-compatibility will cause the genotypes to move to a radically different set of equilibrium frequencies, involving the extinction of incompatibility alleles whose genotypes tend not to cross well with the genotypes that are common in the perturbed system.

When incompatibility alleles are lost in this manner, one can find a set of conditions under which the modelled population evolves to predominantly or exclusively two genotypes. These genotypes are $I_{1} I_{1}$ and $I_{1} I_{3}$, where $I_{3}$ is dominant over $I_{1}$. This is the distyly pattern. The numerical values of the parameters that produce the evolution of the distyly pattern are no doubt model specific and not meant to be taken seriously as such. The general conclusion is that the favoured new mutation $P^{*}$ linked to one of the incompatibility alleles must be strongly favoured and linked tightly with little effective recombination. Thus the model suggests that tight linkage between the $I$ locus and another locus, called $P$ in the model, preceded the evolution of the distyly supergene. Recombination between these two must already have been at the level observed in the supergene, i.e., $10^{-3}$ or less (B. and D. Charlesworth, 1979). Perhaps they occurred together in a situation of suppressed crossover, an inversion, for example. This site in the genome may then have been the focus to which the other loci of the supergene became linked as the distyly syndrome built.

In the model, fitnesses between 1.4 and 2.0 were high enough to shift the incompatibility genotypes to predominantly but not exclusively $I_{1} I_{1}$ and $I_{1} I_{3}$. Then these two genotypes formed 79 per cent to 94 per cent of the population in the cases examined. At these levels a third to a half of randomly assorted pollen reaches incompatible stigmas. Morphological characters that tend to cause appropriately disassortative pollination may thus evolve. For this reason I suggest that the evolution of distyly does not require the presence of exactly two mating types, though eventually the rare $I$ allele must be eliminated either selectively or randomly.

$P^{*}$, although favoured, may not go to fixation when dominant or incompletely dominant. Its failure to recombine with some of the incompatibility alleles that are maintained in the population by favourable crosscompatibilities prevents its fixation in those cases. The frequency (less than $1.0)$ that $P^{*}$ reaches is, in each case, a quasi-equilibrium. It is unstable in the sense that, should effective recombination occur linking $P^{*}$ to a new allele, the frequency of $P^{*}$ would rise rapidly as the new linkage group $P^{*} I_{i}$ replaced $P I_{i}$. But there is no question that this quasi-equilibrium is an equilibrium in the sense that it remains unchanged for many hundreds of generations if effective recombination does not occur. All the quasiequilibria reported in table 3 were maintained unchanged for at least 800 generations. In a real population that is probably enough time for the morphological characters of distyly to begin to evolve and to become linked to the $P-I$ loci. This could make effective recombination even less likely, 
so that the entire distyly pattern may evolve without $P^{*}$ ever reaching fixation.

The reason $P^{*}$ may fail to reach fixation is that the new recombinants $P^{*} I_{i}$ may fail repeatedly to become established in the population. As with the spread of a favourable new mutation (Ewens, 1969), two parameters contribute to the likelihood of establishment of a new recombinant-the probability of random death of rare recombinants and the selective advantage of the rare recombinants. The first, the probability of random death, is by definition somewhat high, since we are only interested in those cases where $I$ alleles can be lost, and that requires a rather high probability of random death. To some extent, then, we have the quandary that the conditions that allow the loss of $I$ alleles also tend to prevent the fixation of $P^{*}$. The second parameter, the selective advantage of the rare recombinant, is clearly important in that a stronger selective advantage will "pull" the rare recombinant to a higher frequency faster, increasing the chances that the recombinant will reach the frequency above which it is no longer subject to random extinction. This explains why $P^{*}$ 's failure to reach fixation is common when $P^{*}$ is dominant, rarer when $P^{*}$ is incompletely dominant and does not occur when $P^{*}$ is recessive. In particular, when $P^{*}$ is dominant and the new recombinant $P^{*} I_{i}$ almost always finds itself in a $P^{*} P^{*}$ genotype, the $P^{*}$ that the new recombinant brought to that genotype does not increase the genotype's fitness. Thus the recombinant's selective advantage is quite low, it does not increase in frequency very rapidly and the period in which it is subject to random loss is extended. When $P^{*}$ is incompletely dominant, the new recombinant $P^{*} I_{i}$ brings some selective advantage to any genotype relative to the non-recombinant $P I_{i}$. That selective advantage tends to "pull" the new recombinant out of the low frequencies where it is in danger of random extinction, and indeed it usually succeeds in doing so. In the incomplete dominance case, the only recombinant that fails to become established is $P^{*} I_{4}$ (table 3 ). $I_{4}$ is maintained at low frequencies because of its cross-compatibility relationships, which means recombinational events are rarer and so the recombinant frequency has to increase further to get out of the range where it becomes extinct randomly. Its selective advantage often does not act fast enough to establish it. When $P^{*}$ is recessive, the new recombinant $P^{*} I_{i}$ greatly increases the fitness of the genotype $P^{*} I_{1} P^{*} I_{i}$. Because $P^{*} I_{1}$ is common, this genotype is also relatively common and its high fitness rapidly increases the frequency of the new recombinant. Thus $P^{*}$ always recombines effectively with surviving $I$ alleles when $P^{*}$ is recessive. Effective recombination may not, however, occur fast enough to prevent the loss of some $I$ alleles.

What is the $P$ locus? There is no theoretical reason why it has to be related to distyly, though it does have to be located, perhaps unrecognized, in the supergene. When the distyly pattern evolves in the three cases examined $-P^{*}$ dominant, incompletely dominant and recessive $-P^{*}$ either goes to fixation or to a quasi-equilibrium frequency at which it is present entirely or almost entirely in the $P^{*} P^{*}$ and $P^{*} P$ genotypes; this mostly occurs when $P^{*}$ is dominant. Thus there is either no longer any genetic variation present at the $P$ locus or genetic variation is seldom observable, since the mating system tends to prevent the $P^{*} P \times P^{*} P$ cross that would generate the $P P$ homozygote. Both these situations make it unlikely that the $P$ locus would be recognized as part of the supergene. Other possible 
actions of the $P^{*}$ mutation, for example selection at the haploid level, have not been modelled here. They are the subject of further work.

Natural populations of sporophytically incompatible plants have more, perhaps many more, than four incompatibility alleles. There are apt to be several recessive, dominant and independent alleles to any given allele. How this affects the loss-of-allele behaviour described above is under investigation.

It is more parsimonious to argue that one form of self-incompatibility, distyly, arose from another, pre-existing, form of self-incompatibility than to argue that distyly arose from self-compatibility. I believe the most important reason that the latter has been the preferred scheme is the lack of a mechanism by which a multiallelic system could change to a diallelic one. This study suggests such a mechanism. Two authors have, however, discounted the loss-of-alleles hypothesis for reasons not considered by the above model. Ganders (1979) states: "In a typical population of a species with multiallelic self-incompatibility, any plant can be fertilized by pollen from almost every other plant in the population. With distyly, however, pollen from half the plants in the population is not compatible on any particular stigma . . . Presumably, then, the ancestors of heterostylous plants did not have an evolutionary choice between diallelic or multiallelic selfincompatibility systems, or the multiallelic one would have been selected . . . a diallelic system would not be expected to evolve from a more efficient multiallelic one."

He assumes that selection favours population mating efficiency. This is not assumed in the above model. Further, it is a group selection hypothesis, and as such $I$ assume is subject to the general restrictions placed upon a group selection hypothesis and that it is weak compared to any reasonable individual selection hypothesis that could be put forward (Lewontin, 1970). Ganders may have meant to include in his statement the concept that individual selection for maximal cross-compatibility is normally expected to act against the loss of incompatibility alleles. The model above shows the limitations of that expectation, and in particular how selection for maximal cross-compatibility can act in conjunction with other forces to drive incompatibility alleles extinct, leaving a distyly-type diallelic incompatibility system.

The Charlesworths (1979) have stated a second objection to the loss-ofalleles hypothesis. They cite the observation of homostyles, derived by crossover in the distyly supergene, that are self-compatible, because the pistil has an active pin reaction and the pollen an active thrum reaction. They claim that incompatibility loci as found in homomorphic taxa cannot explain this pattern-therefore incompatibility in distyly must not be controlled by a homomorphic-type locus, so that distyly cannot have evolved from a homomorphic-type incompatibility system. I have discussed this elsewhere (Muenchow, 1981). The genetic structure and physiology of incompatibility in distyly are largely unknown, and that in homomorphic taxa still sketchy. It is, however, possible, by drawing a picture of a hypothetical distyly supergene that includes an incompatibility locus as described from homomorphic taxa, to derive by crossover the homostyle with the observed properties. Several other phenomena related to crossover in the distyly supergene are also explained. 
The loss-of-alleles hypothesis for the evolution of distyly has been unpopular because no one has offered a mechanism for the loss of incompatibility alleles other than the implausible suggestion of random extinction. The above model shows that the ever-present selection for maximal crosscompatibility can, under certain circumstances, selectively remove incompatibility alleles in such a way that the remaining alleles exhibit the distyly pattern of dominance/recessiveness. That this may be related to the evolution of distyly is encouraged by the fact that an important restriction on the model, very tight linkage, is observed in distyly. I hope here to put the loss-of-alleles hypothesis back into the arena of discussion.

Acknowledgments.-I am deeply indebted to Jane Bock for making the University of Colorado computer facilities available to me. I also wish to thank Jane, Richard Hopkins, Michael Grant, Meredith Lane, Yan Linhart, Jeff Mitton and a reviewer for many helpful comments on the manuscript.

\section{REFERENCES}

BAKER, H. G. 1966. The evolution, functioning and breakdown of heteromorphic incompatibility systems. Evolution, 20, 349-368.

CHARLESWORTH, B., AND CHARLESWORTH, D. 1979. The maintenance and breakdown of distyly. Amer. Nat., 114, 499-513.

CHARLESWORTH, D., AND CHARLESWORTH, B. 1979. A model for the evolution of distyly. Amer. Nat., 114, 467-498.

CROWE, L. K. 1964. The evolution of outbreeding in plants. I. The angiosperms. Heredity, $19,435-457$.

DE NETTANCOURT, D. 1977. Incompatibility in Angiosperms. Springer-Verlag, Berlin.

EWEN, w. J. 1969. Population Genetics. Methuen and Co., Ltd, London.

GANDERS, F. R. 1979. The biology of heterostyly. N.Z. Jour. of Bot., 17, 607-635.

LEWIS, D. 1954. Comparative incompatibility in angiosperms and fungi. Adv. Genet., 6, 235-285.

LEWOnTIN, R. 1970. The units of selection. Ann. Rev. Genetics, 1, 1-20.

MUENCHOW, G. 1981. An S-locus model for the distyly supergene. Amer. Nat., 118, 756-760.

VUILLEUMIER, B. S. 1967. The origin and evolutionary development of heterostyly in the angiosperms. Evolution, 21, 210-226.

WRIGHT, S. 1939. The distribution of self-sterility alleles in populations. Genetics, 24, 538-552. 